\title{
ISSUES IN MEDICINE \\ Achieving the HIV and AIDS National Strategic Plan: A practical calculator for local target setting in district health facilities
}

\author{
Nicholas G Leydon, Francois Venter, Patricia D Webster, Winnie Moleko, Regina Osih, Pierre M Barker
}

The South African HIV National Strategic Plan (NSP) aims to provide access to appropriate treatment, care and support to $80 \%$ of the HIV-infected population by $2011 .{ }^{1}$ By mid-2008, highly active antiretroviral treatment (HAART) was being dispensed to about half the HIV-infected population in need. ${ }^{2}$ Reaching the NSP targets will require full mobilisation of all of South Africa's health facilities. While the NSP has broad political and programmatic support from the Department of Health and civil society, and managers are able to recite the national targets, it has been difficult for these managers to relate the targets to their own geographical areas of responsibility. National, regional and district targets for HIV care have been set from South Africa's relatively good census, modelling and epidemiological data. ${ }^{3}$ However, few practical tools are available to help clinicians and managers understand their facility's actual contribution to the district regional and national NSP targets for each step of the HIV care pathway (HIV testing, CD4 testing, HAART referral and initiation). The calculation of HAART initiation targets is complicated by the anticipated additional demand for treatment that will be generated by a change in the recommended CD4-count threshold for initiation of treatment. ${ }^{4}$ Accordingly, we provide a data-based tool that is readily available, and that district and facility managers can use to calculate their annual steady-state HIV testing, CD4 testing and HAART initiation requirements. These calculated values can be used for local and regional planning and to assess and improve current performance at facility level.

Previous tools have provided districts with the ability to calculate their estimated monthly need for HAART initiations ${ }^{5}$ using statistics from the Actuarial Society of South Africa (ASSA). ${ }^{3}$ Because many primary health clinics (PHCs) do not yet initiate patients on HAART, they need a way to relate their diagnostic and staging activities to the initiation targets set for the district. A simple tool can be used to calculate the targets for each step in the treatment pathway, using patient visit data collected in the Department of Health Information System

Nicholas G Leydon and Patricia D Webster are based at the Institute for Healthcare Improvement, Cambridge, MA, USA; Francois Venter, Winnie Moleko and Regina Osih at the Reproductive Health and HIV Research Unit, Johannesburg; and Pierre M Barker at the Institute for Healthcare Improvement and the University of North Carolina, Chapel Hill, NC, USA.
(DHIS) and district HAART initiation estimates derived from ASSA data. ${ }^{3}$ These targets can then be compared with current performance and used to identify areas where improvement is needed.

An estimate of the number of clients in a district who would require HIV and CD4-count testing to identify all those who need HAART (100\% coverage, calculated from Barker and Venter ${ }^{5}$ ) in that area can be calculated from the prevalence of HIV among the antenatal-care population (approximately 29\% in this example district), and measured proportion of HIVpositive clients with CD4 counts $<200$ cells $/ \mu l$ (approximately $30 \%$ in this district - Fig. 1A). These calculations provide broad targets that are useful for district planners; however, since each health facility in a district represents only a fraction of that district's total HAART need, further breakdown is required to calculate facility-level targets.

Using the DHIS reports of monthly head count, managers can calculate each PHC's contribution to the total district headcount. Fig. 1B shows how this breakdown can provide the number of patients who must complete each step of HIV care every month in all clinics to achieve a district goal of 656 HAART initiations. For example, PHC 8 represents $6 \%$ of the district head count and, assuming equal distribution of HIV prevalence across the district, should refer approximately 39 clients per month for initiation ( $6 \%$ of the district goal of 656 clients requiring initiation). The clinic must first counsel, test and prepare sufficient clients at each step to yield this number of HAART referrals. Using the same historical data for antenatal HIV prevalence and CD4 count in the district, PHC 8 can calculate back through these steps to derive the number of clients it needs to counsel for HIV testing (446 patients per month). A simple tool for calculating district-wide and facilityspecific targets for HIV testing and HAART initiations is available at www.ihi.org/topics/developingcountries.

These calculations assume a well-integrated health system for HIV testing, CD4 testing, HAART referral and initiation that functions at $100 \%$ efficiency. In reality, many clients are lost at each step of the testing, referral and initiation care pathway owing to system inefficiencies. South Africa's HAART initiation targets will not be reached unless these inefficiencies are addressed. Using methods to improve the reliability of the HAART preparation and initiation processes, ${ }^{6}$ facility-specific numerical targets have been used in the Eastern Cape's O R Tambo District by PHC sites to increase the number of patients available for ARV initiation. Before introducing this tool, most facilities operated far below the estimated number of clients needed to reach the HAART initiation targets. Once facilities committed to reaching local testing and referral targets, they developed local strategies to provide voluntary counselling 


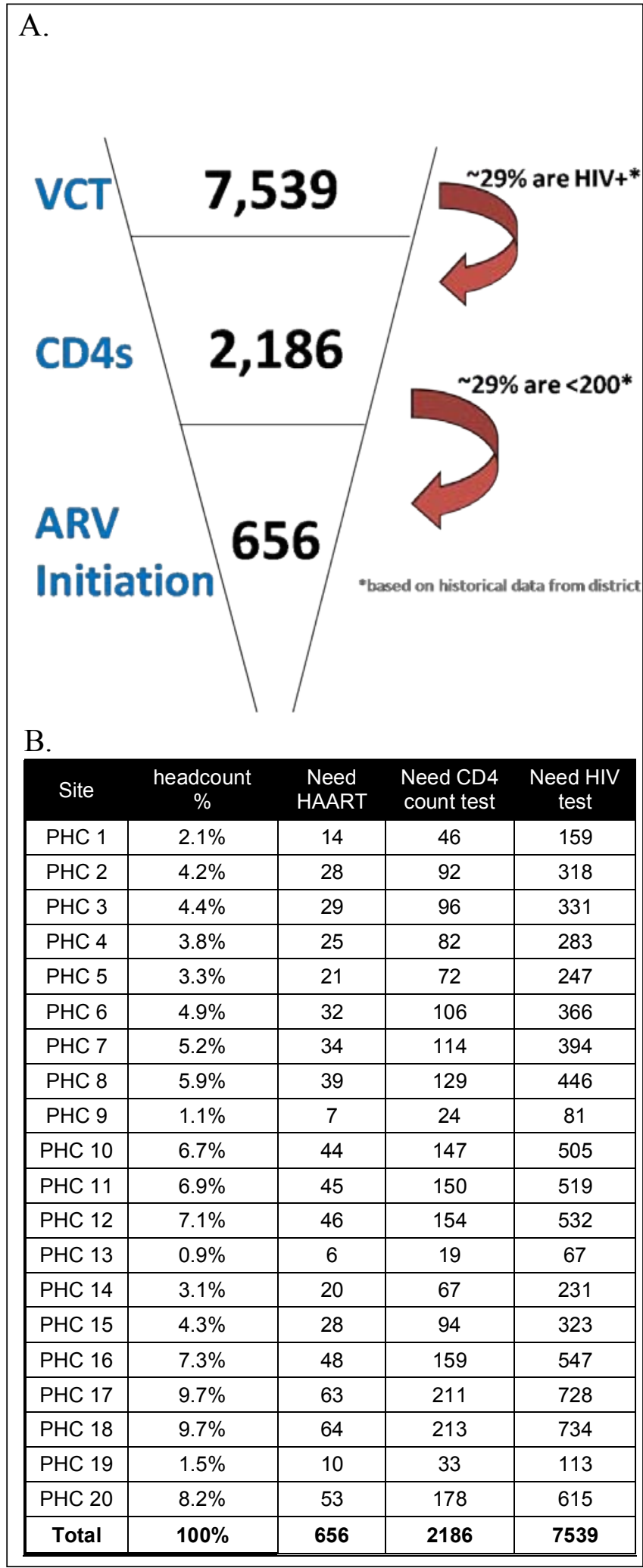

Fig. 1A. Calculation of number of clients who will need HIV and CD4count testing in a district with 20 PHCs, in which an estimated 656 new HAART initiations are required each month. Fig. 1B. Facility-level HIV and CD4 test estimates derived from 'District HIV target calculator' (www.ihi.org) for a district that initiates 656 new clients on HAART each month, where HIV prevalence for the population is $29 \%$, and $30 \%$ of HIV-positive clients have a CD4 less than $200 \mathrm{cell} / \mathrm{\mu l}$. Calculations for each health care site: 'Headcount \%' is from district access figures, 'Need HAART' is (656 × 'headcount \%'), 'Need CD4-count test' is ('Need HAART' $\div 30 \%$ ), and 'Need HIV test' is ('Need CD4' $\div 29 \%$ ). and testing (VCT) for every antenatal client and tuberculosis (TB) suspect. HIV testing in the Mhlontlo sub-district doubled from 1500 /month to $>3000 /$ month over a 6 -month period after these local VCT targets were introduced at district health facilities.

The South African health system may adopt new guidelines related to ARV initiation based on recommendations from the World Health Organization (WHO) to start eligible patients at $\mathrm{CD} 4<350 / \mu \mathrm{l} .{ }^{4} \mathrm{~A}$ change in CD4 criteria will create an immediate increase in the pool of patients eligible to start HAART. However, once this backlog has been worked down, the system will return to a steady state, and the number of patients eligible for treatment each year, as predicted by this model, will remain unchanged, because the annual influx of new patients requiring HAART is determined by the annual rate of new infections in previous years. On average, a cohort of patients infected with HIV each year ( 500 000 in South Africa $^{3}$ ) will take 8 years to require HAART initiation if the threshold is 200 cells $/ \mu \mathrm{l}$, and 6 years if the threshold is 350 cells / $\mu 1 .{ }^{7}$ Raising the CD4-count level at which patients become eligible for HAART will simply shorten the interval from infection to HAART treatment, and not increase the annual pool of patients requiring HAART (Fig. 2). The change in CD4count threshold for HAART will, however, create a temporary backlog of approximately 1 million patients (in South Africa's case, the national HAART initiation requirement in 2010 will increase from 500000 to 1.5 million). After elimination of the backlog, the initiation requirement in subsequent years will revert to a steady state of about 500000 /year. Given that the current performance for HAART initiation is well below the annual need, it is likely that the system will take some years to respond to the surge in demand created by the new guidelines. The calculator can be used to estimate the initiations and HIV/ CD4 testing requirements associated with the steady state. The change in CD4 threshold will create a one-time increase in demand for initiation and associated HIV/CD4 testing that is equivalent to double the annual steady-state requirements.

Health facilities across South Africa are being asked to improve services to achieve targets for the NSP, the Millennium

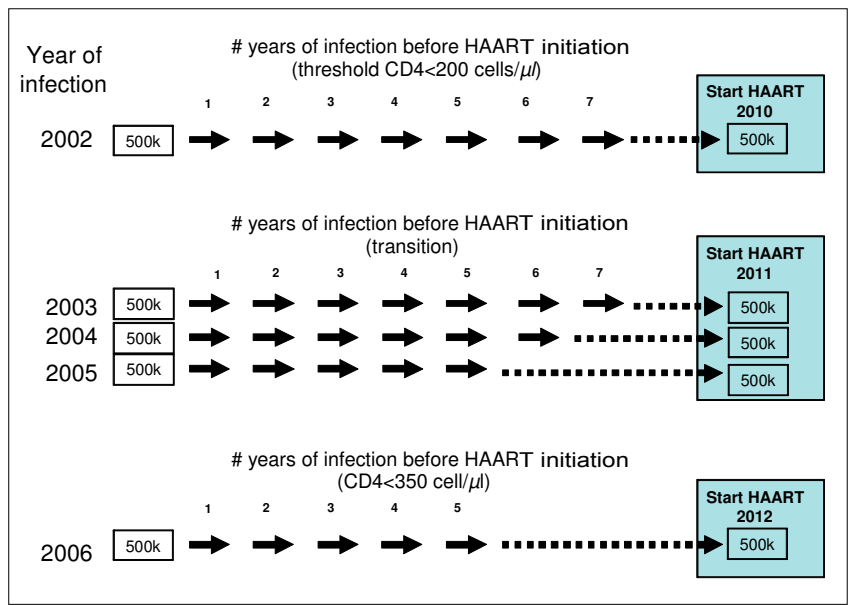

Fig. 2. Estimated number of new patients requiring HAART initiation in South Africa in 2010 (based on CD4 threshold of $200 \mathrm{cell} / \mathrm{\mu l}$ ), 2011 (during transition to new threshold of $350 \mathrm{cells} / \mathrm{\mu l}$ ), and 2012, assuming that the backlog of patients requiring HAART created by the new CD4 threshold recommendations ${ }^{4}$ could be cleared in 2011. 
Development Goals or other campaigns. While facilitylevel staff are often urged to 'improve' performance, they have difficulty assessing quantitatively how their facility should contribute to the intended outcome of each strategic initiative. The tool uses data that are readily available to district managers for the purpose of setting targets, measuring progress and optimising resources at each facility.
2. Geffen N. HAART coverage and unmet need in South Africa. HIV Treat Bull 2009; 10(5/6): 26. 3. Actuarial Society of South Africa. ASSA2003. http://aids.actuarialsociety.org.za/ASSA2003Model-3165.html (accessed 2 December 2009).

4. World Health Organization. Rapid Advice: Antiretroviral Therapy for HIV Infection in Adults and Adolescents. 30 Nov 2009. http://www.who.int/hiv/pub/arv/advice/en/index.html (accessed 12 January 2010).

5. Barker P, Venter F. Setting district-based annual targets for HAART and PMTCT - a first step in planning effective intervention for the HIV/AIDS epidemic. S Afr Med J 2007; 97(10): 916917.

6. Barker PM, McCannon CJ, Mehta N, et al. Strategies for scale-up of antiretroviral treatment in South Africa through health system optimization. J Infect Dis 2007; 196 Suppl 3: S457-463.

7. Pantaleo G, Graziosi C, Fauci AS. New concepts in the immunopathogenesis of human immunodeficiency virus infection. N Engl J Med 1993; 328: 327-335.

\title{
CLINICAL IMAGES
}

\section{Reversible nephrotic syndrome secondary to pulmonary hydatid disease}

\author{
Tahar Gargah, Rim Goucha-Louzir, Youssef Gharbi, Rachid Mohamed Lakhoua
}

Most patients with pulmonary hydatidosis are children. The disease may be asymptomatic or revealed by unusual events such as a glomerulopathy. ${ }^{1}$

\section{Case report}

An 8-year-old boy from a rural part of Tunisia presented with generalised oedema and macroscopic haematuria. There was no familial history of renal disease. He had a normal blood pressure $(100 / 60 \mathrm{mmHg})$, and a pleural effusion was detected. Urinalysis showed nephrotic range proteinuria ( $375 \mathrm{mg} / \mathrm{kg} / \mathrm{d}$ ) and microscopic haematuria. His serum total protein concentration was $40 \mathrm{~g} / \mathrm{l}$ and his serum albumin was $10 \mathrm{~g} / \mathrm{l}$. Renal biopsy showed capillary wall thickening and duplication, and mesangial cell proliferation in the glomeruli, characteristic of mesangiocapillary glomerulonephritis. Renal and abdominal ultrasound images showed increased echogenicity of the kidneys and mild ascites. Radiology revealed three large pulmonary hydatid cysts (Figs 1 and 2). The largest cyst occupied the entire right upper lobe and compressed the superior vena cava. Hydatid disease was confirmed by a strongly positive serum enzyme-linked immunosorbent assay (ELISA) for echinococcus.

The patient was treated with high protein intake, dipyramidol and captopril; both right lung cysts were resected,

Tahar Gargah, Rim Goucha-Louzir and Rachid Mohamed Lakhoua are based at the Charles Nicolle Hospital in Tunis, Tunisia, and Youssef Gharbi in the Department of Paediatric Surgery at the Habib Thameur Hospital in Tunis.

Corresponding author: Tahar Gargah (kitomora@yahoo.fr)

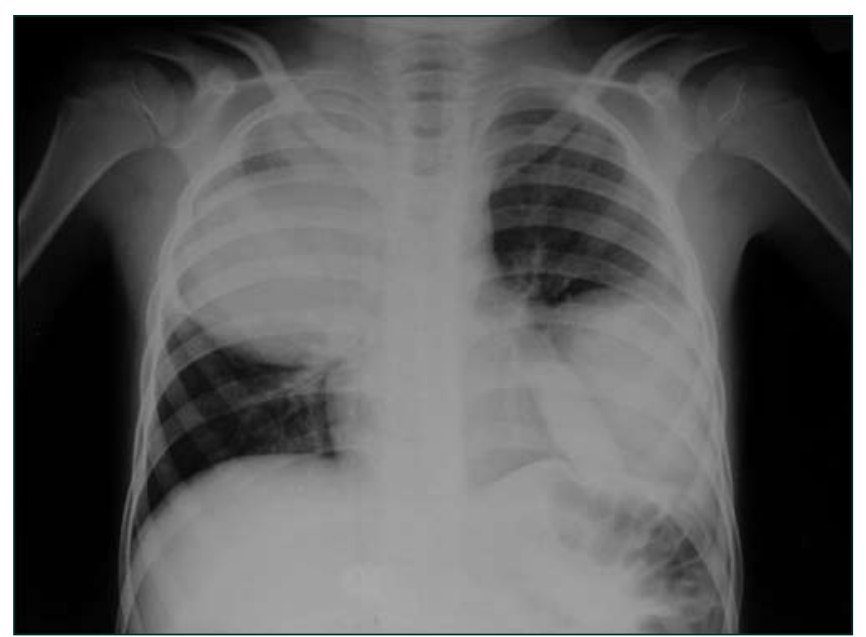

Fig. 1. Chest radiograph showing a bilateral well-circumscribed dense lesion.

followed by the left pulmonary cyst 4 weeks later. Hydatid cyst was confirmed histopathologically. He recovered well, the serum ELISA for echinococcus became negative, and followup urine examination and thoracic computerised tomography were normal 6 months after surgery, confirming good renal recovery and absence of pulmonary hydatid disease.

\section{Discussion}

Renal involvement during hydatid disease is well recognised. ${ }^{1}$ The most common manifestation is proteinuria, with or without nephrotic syndrome. Several histopathological types have been demonstrated..$^{1-3}$ The pathogenesis of glomerular disease in patients with hydatid disease is not well understood, with most supporting an immune complex-mediated mechanism. Echinococcal antigen and corresponding antibody in the glomeruli have been demonstrated by immunoperoxidase studies. ${ }^{4}$ The site of 